\title{
NOUVELLE
}

\section{L'essai de proximité aux méthionines: une approche pour glaner les structures des récepteurs}

Martin Clément, Emanuel Escher

\author{
Département de Pharmacologie, \\ Faculté de Médecine, \\ Université de Sherbrooke, \\ $3001,12^{\mathrm{e}}$ Avenue Nord, Sherbrooke (Québec), J1H 5N4 Canada. \\ Martin.Clement@USherbrooke.ca \\ Emanuel.Escher@USherbrooke.ca
}

> Les récepteurs couplés aux protéines $G$ (GPCR) sont les récepteurs membranaires les plus nombreux et représentent la cible de plus de la moitié des médicaments. En étudiant leur structure tridimensionnelle, on pourrait créer de nouveaux médicaments et identifier de nouvelles avenues thérapeutiques. Malheureusement, les connaissances concernant la structure des GPCR sont limitées et il est actuellement très difficile d'obtenir une quantité et une pureté appréciables d'un récepteur. Jusqu'à présent, par cristallographie, la structure d'un seul GPCR, la rhodopsine bovine [1], a été directement obtenue. Les éléments structuraux de l'interaction ligand-récepteur sont donc souvent déterminés soit uniquement par modélisation moléculaire par homologie avec la structure de la rhodopsine bovine, soit en y incorporant des résultats de mutagenèse dirigée et/ou ceux des études de structure-fonction. Toutes ces méthodes sont indirectes.

Le marquage par photoaffinité constitue une approche directe pour identifier les points de contact ligand- récepteur. Cette approche utilise un analogue du ligand naturel contenant un résidu photosensible qui, par suite de l'activation par la lumière, peut former une liaison covalente avec son environnement immédiat dans la pochette de liaison du récepteur (Figure 1). Ce site de photomarquage représente une région de contact entre le ligand et le récepteur et peut être identifié par une série de digestions enzymatiques et/ou chimiques [2] ou par spectrométrie de masse [3]. Cette approche a été utilisée avec succès dans le cas de plusieurs récepteurs, en particulier les GPCR [4], incluant les récepteurs de l'angiotensine II (Ang II) $[5,6]$. Pour le récepteur du sous-type $A T_{1}$, les points de contact de l'Ang II se situent dans la deuxième boucle extracellulaire et dans le septième domaine transmembranaire du récepteur [6-8]. Cette information nous a permis de déduire l'orientation du ligand dans la pochette de liaison du récepteur et de proposer un modèle moléculaire du récepteur $\mathrm{AT}_{1}$ [6]. Une situation semblable a également été mise en évidence pour le récepteur du sous-type $\mathrm{AT}_{2}$ [5].

Au cours des études de photomarquage, nous avons observé que l'acide aminé photoactivable para-benzoyl$L$-phénylalanine (Bpa) présentait une sélectivité prononcée pour la méthionine (Met). Cette observation a été confirmée par de nombreuses études identifiant une méthionine comme résidu de contact entre un ligand (contenant le Bpa) et son récepteur. Les études de photochimie révèlent que la benzophénone, le chromophore contenu dans le Bpa, possède dans sa forme activée une forte sélectivité pour le groupe thioéther [9] présent uniquement dans la Met (Figure l). Nous avons exploité cette sélectivité du Bpa pour mettre au point une nouvelle approche: l'essai de proximité aux méthionines (MPA). Afin de déterminer l'environnement tridimensionnel de la position carboxy-terminale de l'angiotensine II dans le récepteur $A T_{1}$, nous avons systématiquement remplacé par des Met tous les résidus du récepteur qui pouvaient se trouver dans cet environnement de contact. Lorsque les récepteurs mutants sont photomarqués, le ligand photosensible réagit avec la Met si elle se trouve à proximité du résidu Bpa, au détri-
Figure 1. Schéma réactionnel de la photoactivation du Bpa sur un résidu méthionine et les produits de clivage par suite d'une digestion au $\mathrm{CNBr}$. 
ment du résidu normalement photomarqué dans le récepteur natif. Ce fait se manifeste clairement avec un traitement de protéolyse chimique, puisque le bromure de cyanogène $(\mathrm{CNBr})$ clive spécifiquement les liens peptidiques carboxyterminaux des méthionines. Ce clivage donne naissance à un fragment différent de celui obtenu avec le récepteur natif (Figure 2). Le mérite du MPA réside dans le fait qu'une seule étape de digestion chimique est nécessaire pour identifier dans le récepteur un nouveau point de contact avec le ligand.

Nous avons construit des mutants du récepteur $A T_{1}$ en substituant contre une Met certains des résidus des domaines transmembranaires 3,6 et 7 , qui forment la pochette de liaison. L'essai MPA avec ces mutants a été effectué avec un photoligand radiomarqué contenant le Bpa dans la $8^{e}$ position: ${ }^{125} \mathrm{I}-\left[\mathrm{Sar}^{1}, \mathrm{Bpa}^{8}\right]$ Angll. Nous avons ainsi mis en évidence neuf nouveaux points de contact pour la position 8 de l'Ang II [10]. L'incorporation de ces points de contact dans la modélisation par homologie du récepteur permet de réduire les structures possibles et ainsi de proposer une structure tridimensionnelle du récepteur $\mathrm{hAT}_{1}$ en présence de son ligand. Cette structure suggère fortement que les domaines transmembranaires 3,6 et 7 contribuent à l'interface de reconnaissance ligandrécepteur et participent à la pochette de liaison du ligand.

En conclusion, le MPA est une technique puissante qui permet de déterminer expérimentalement la nature des résidus formant une partie de la pochette de liaison du récepteur $\mathrm{hAT}_{1}$ de l'angiotensine II et, par conséquent, des parties importantes de la structure du

récepteur.

Puisque, de façon générale, la mutation d'un a c i d e a $m$ i n é endogène en méthionine cause rarement des pertes d'affinité du récepteur, I'approche MPA peut être appliquée à d'autres récepteurs peptidergiques afin de raffiner notre connaissance des interactions ligand-récepteur pour une multitude de récepteurs et finalement elle permet de mener à la création de nouveaux agents thérapeutiques. $\diamond$

The methionine proximity assay:

an approach to glean

receptor structures

\section{RÉFÉRENCES}

1. Palczewski K, Kumasaka T, Hori T, et al. Crystal structure of rhodopsin: A G protein-coupled receptor. Science $2000 ; 289$ : 739-45.

2. Servant G, Dudley DT, Escher $\varepsilon$, Guillemette G. Analysis of the role of $\mathrm{N}$-glycosylation in cell-surface expression and binding properties of angiotensin Il type-2 receptor of rat pheochromocytoma cells. Biochem J 1996 ; 313 : 297-304.

3. Sachon $\varepsilon$, Bolbach G, Lavielle S, et al. Met174 side chain is the site of photoinsertion of a substance $P$ competitive peptide antagonist photoreactive in position 8. FEBS Lett 2003 ; $544: 45-9$.

4. Dong $M$, Wang $Y$, Pinon DI, et al. Demonstration of a direct interaction between residue 22 in the carboxylterminal half of secretin and the amino-terminal tail of the secretin receptor using photoaffinity labeling. J Biol Chem 1999 ; 274 : 903-9.

5. Servant G, Laporte SA, Leduc R, et al. Identification of angiotensin II-binding domains in the rat AT2 receptor with photolabile angiotensin analogs. J Biol Chem 1997 ; $272: 8653-9$.

6. Laporte SA, Boucard AA, Servant G, et al. Determination of peptide contact points in the human angiotensin II type I receptor (ATI) with photosensitive analogs of angiotensin II. Mol Endocrinol $1999 ; 13$ : 578-86.

7. Boucard AA, Wilkes BC, Laporte SA, et al. Photolabeling identifies position 172 of the human AT(1) receptor as a ligand contact point: receptorbound angiotensin II adopts an extended structure. Biochemistry $2000 ; 39$ : 9662-70.

8. Perodin J, Deraet M, Auger-Messier M, et al. Residues 293 and 294 are ligand contact points of the human angiotensin type 1 receptor. Biochemistry 2002 ; $41: 14348-56$.

9. Bobrowski K, Marciniak B, Hug GL. 4-Carboxybenzophenone-sensitized photooxidation of sulfur-containing amino acids. Nanosecond laser flash photolysis and pulse radiolysis studies. J Am Chem Soc 1992 ; 114 : 10279-88.

10. Clément M, Martin SS, Beaulieu ME, et al. Determining the environment of the ligand binding pocket of the human angiotensin II type I ( $\mathrm{hAT}_{1}$ ) receptor using the methionine proximity assay. J Biol Chem 2005 ; $280: 27121-9$. 